\title{
UNTER- UND OBERFUNKTIONEN UND DIFFERENTIALUNGLEICHUNGEN ZWEITER ORDNUNG IN NORMIERTEN RÄUMEN
}

\author{
ROLAND LEMMERT
}

\begin{abstract}
An invariance theorem for a cone in a normed linear space with respect to solutions of general elliptic inequalities is obtained, which includes and generalizes monotonicity theorems for systems of second order ordinary, parabolic and elliptic differential inequalities.
\end{abstract}

1. Einleitung. Differentialungleichungen und Monotoniesätze spielen bei Randwertproblemen für elliptische, parabolische und gewöhnliche Differentialgleichungen zweiter Ordnung eine zentrale Rolle. In neuerer Zeit wurden Monotoniesätze und verschiedene Maximumprinzipien für Systeme solcher Gleichungen mit Erfolg als Invarianzprobleme für gewisse Ordungskegel oder Kugeln (oder allgemeiner für konvexe Mengen) aufgefasst, vgl. etwa Weinberger [15] und Redheffer und Walter [10].

Fountain und Jackson [2] (für eine Gleichung) und Heimes [3] beweisen Monotoniesätze für gewöhnliche Differentialungleichungen, in denen die zweiten Ableitungen durch allgemeinere Differentialquotienten ersetzt werden, vgl. auch das Buch von Bernfeld und Lakshmikantham [1].

Ziel der vorliegenden Note ist es, die bekannten Ergebnisse über Differentialungleichungen zweiter Ordnung in einem normierten Raum $E$ unter einem einheitlichen Gesichtspunkt zu erfassen und zu erweitern. Die Verallgemeinerungen betreffen dabei sowohl die zulässigen Räume, in denen die Lösungen ihre Werte annehmen, als auch die verwendeten Ungleichungsund Lösungsbegriffe. Dabei bedienen wir uns reiner Differentialungleichungsmethoden, vermeiden also nach Möglichkeit Existenzsätz für die behandelten Gleichungen und kommen so ohne Regularitätsbedingungen etwa an das Grundgebiet, die Koeffizienten der betrachteten Gleichungen oder den normierten Raum $E$ aus.

Monotoniesätze für gewöhnliche Differentialgleichungen in $l_{\infty}(Z)$ wurden z.B. von Thompson [12] bei der Anwendung der Rothe-Methode auf elliptische Differentialgleichungen in einem unendlichen Streifen verwendet.

2. Bezeichnungen. Es sei $\Omega \subset \mathbf{R}^{n}$ offen, $\Gamma \subset \partial \Omega, A(x)$ für jedes $x \in \Omega$ eine symmetrische, positiv semidefinite $n \times n$-Matrix, $T(x)$ ein linearer Teilraum des $\mathbf{R}^{n}$ und $b(x) \in \mathbf{R}^{n}$; die Funktionen $a_{0}, a_{1}: \Gamma \rightarrow[0, \infty)$ seien vorgegeben

Received by the editors August 19, 1977.

AMS (MOS) subject classifications (1970). Primary 35B45. 
und jedem $x \in \Gamma$ eine äussere Normale $\nu(x)$ im Sinne von Walter [14, IV.31.I] zugeordnet. Für die in einer Umgebung eines Punktes $x \in \Omega$ nach oben beschränkte reelle Funktion $\eta$ setzen wir

$$
\begin{array}{r}
\left(\bar{D}_{A}^{2} \eta\right)(x)=\limsup _{t \rightarrow 0} \sup \left\{\frac { 1 } { t ^ { 2 } } \sum _ { i = 1 } ^ { n } \left(\eta\left(x+t A^{1 / 2}(x) e_{i}\right)-2 \eta(x)\right.\right. \\
\left.+\eta\left(x-t A^{1 / 2}(x) e_{i}\right)\right):
\end{array}
$$

$\left(e_{i}\right)_{1}^{n}$ ist Orthonormalsystem im $\left.\mathbf{R}^{n}\right\}$

$\left(A^{1 / 2}(x)\right.$ ist die positiv semidefinite Wurzel aus $\left.A(x)\right), D_{A}^{2} \eta$ entsprechend für nach unten beschränktes $\eta$ mit lim inf und inf und $D_{A}^{2} \eta=\bar{D}_{A}^{2} \eta=D_{A}^{2} \eta$, falls die beiden letzteren Limites existieren und gleich sind.

Ist $\eta$ im Punkt $x$ zweimal differenzierbar, so gilt (in kartesischen Koordinaten)

$$
D_{A}^{2} \eta(x)=\sum_{i, j=1}^{n} a_{i j}(x) \eta_{x_{i} x_{j}}
$$

Sind $X$ und $Y$ zwei beliebige lineare Räume, so ist $L(X, Y)$ der Raum aller linearen Abbildungen von $X$ nach $Y$. Mit $\eta_{T}^{\prime}(x) \in L(T(x), \mathbf{R})$ wird Differentiation nach $T(x)$ an der Stelle $x \in \Omega$ bezeichnet, d.h. es gilt für $h \in T(x)$

$$
\eta(x+t h)=\eta(x)+t \eta_{T}^{\prime}(x) h+o(|t|) \quad(t \rightarrow 0) .
$$

Das Symbol $\eta_{b}^{\prime}(x)$ steht für die Richtungsableitung $\lim _{t \rightarrow 0}\{\eta(x+t b(x))-$ $\eta(x)\} / t$.

Für $x \in \Gamma$ ist $a_{1} \bar{D}_{\nu}$ gemäss

$$
\left(a_{1} \bar{D}_{\nu} \eta\right)(x)=\limsup _{k \rightarrow \infty} a_{1}(x)\left(\eta(x)-\eta\left(x_{k}\right)\right) /\left|x-x_{k}\right|
$$

definiert $\left(\left(x_{k}\right)\right.$ ist die die Normale $\nu(x)$ definierende Folge aus $\Omega$; entsprechendes gilt für $a_{1} \underline{D}_{\nu}$ und $a_{1} D_{\nu}$. Wie üblich sind $\underline{D}^{+}, \bar{D}^{+}, \underline{D}^{-}$und $\bar{D}^{-}$ die vier Dini-Derivierten und

$$
\left(\underline{D}_{b}^{+} \eta\right)(x)=\liminf _{t \rightarrow 0+} \frac{1}{t}\{\eta(x+t b(x))-\eta(x)\}
$$

die rechte untere Dini-Richtungsableitung in Richtung $b(x)\left(\bar{D}_{b}^{+}\right.$ entsprechend).

Mit $E$ bezeichnen wir einen beliebigen reellen normierten Raum mit Norm $\|\cdot\|$ und topologischem Dualraum $E^{*} ; E$ sei durch einen nichtleeren abgeschlossenen Kegel $(K+K \subset K, \lambda K \subset K, \lambda \geqslant 0)$ in $E$ geordnet, und $K^{*}=\left\{\phi \in E^{*}: \phi \geqslant 0\right.$ auf $\left.K\right\}$ sei der zu $K$ duale Kegel $(K \cap-K=\{0\}$ wird nicht verlangt). Ist $T$ ein Teilraum des $\mathbf{R}^{n}$ und $p \in L(T, E), \phi \in E^{*}$, so ist $\phi_{T}(p)$ das durch $\phi_{T}(p)(h)=\phi(p(h))(h \in T)$ definierte Element aus $L(T, \mathbf{R})$.

Für Funktionen $u$ : $\Omega \rightarrow E$ sind obige Ableitungsdefinitionen stets als 
schwache Gâteaux-Ableitungen zu verstehen: So sagen wir z.B., dass $u_{T}^{\prime}(x)$ existiert, wenn für $h \in T$ und $\phi \in E^{*}$

$$
\phi(u(x+t h))=\phi(u(x))+t \phi\left(u_{T}^{\prime}(x)(h)\right)+o(|t|) \quad(t \rightarrow 0)
$$

gilt (d.h. wenn für jedes $\phi \in E^{*}$ die Ableitung $(\phi \circ u)_{T}^{\prime}(x)$ existiert und gleich $\phi_{T}\left(u_{T}^{\prime}(x)\right)$ ist). Entsprechendes gilt für $D_{A}^{2} u, u_{b}^{\prime}$ und $a_{1} D_{\nu} u$.

Ist $\phi \in E^{*}$ mit $\|\phi\|=1$, so nennen wir $H(\phi)=\{x \in E: \phi(x)=0\}$ die durch $\phi$ bestimmte Hyperebene durch Null. Es gilt die Hessesche Abstandsformel

$$
\operatorname{dist}(H(\phi), u)=|\phi(u)| .
$$

Ist $u \in E \backslash K$ und $d(u):=\operatorname{dist}(K, u)$, so existiert nach dem Satz von HahnBanach eine den Kegel $K$ und die offene Kugel $B(u, d(u))$ trennende Hyperebene $H(\phi)$, d.h. es gilt

$$
\phi(z) \geqslant 0>\phi(v), \quad z \in K, v \in B(u, d(u)) .
$$

(Damit sind solche $\phi$ aus $K^{*}$.) Für jede derartige Hyperebene $H(\phi)$ gilt

$$
\operatorname{dist}(K, u)=\operatorname{dist}(H(\phi), u)=-\phi(u) .
$$

Wir definieren folgende Funktionenklasse

$$
\mathcal{E}=\mathcal{E}\left(A, T, b, \Omega, a_{0}, a_{1}, \nu, \Gamma\right):
$$

Die reellwertige Funktion $U=U(x, s, q)$, die für jedes $(x, s) \in \Omega \times(0, \infty)$ auf $L(T, \mathbf{R})$ definiert ist, gehört zur Klasse $\mathcal{E}$, wenn gilt:

Ist $\eta: \bar{\Omega} \rightarrow[0, \infty)$ stetig, ist für $x \in \Omega$ stets $\underline{D}_{A}^{2} \eta>-\infty, \underline{D}_{b}^{+} \eta>-\infty$, ist $a_{0} \eta+a_{1} D_{\nu} \eta \leqslant 0$ auf $\Gamma$ und gilt ausserdem für alle $x \in \Omega$, in denen $\eta$ positiv ist und $\eta_{T}^{\prime}(x)$ existiert, die Ungleichung

$$
\underline{D}_{A}^{2} \eta+\underline{D}_{b}^{+}+U\left(x, \eta, \eta_{T}^{\prime}\right) \geqslant 0
$$

so folgt $\eta \equiv 0$.

BEMERKUNGEN. (1) Ist $\Omega$ beschränkt, $b \equiv 0, \Gamma=\partial \Omega$ und gilt für Lösungen von $D_{A}^{2} \eta \geqslant 0$ das Maximumprinzip, so gehört $U \equiv 0 \mathrm{zu} \mathcal{E}$.

(2) Allgemeinere hinreichende Bedingungen für die Zugehörigkeit von Funktionen $U \mathrm{zu} \mathcal{E}$, die insbesondere parabolischen und gewöhnlichen Gleichungen angepasst sind, findet man in [5]. Ein allgemeines Kriterium für die Zugehörigkeit zu $\mathcal{E}$ geben wir in 4 . an.

3. Ein Invarianzsatz für Kegel. Es sei $g=g(x, z, p)$ für jedes $(x, z) \in \Omega \times$ $E$ eine Funktion von $L(T(x), E)$ nach $E$. Wir betrachten Lösungen $u$ von

$$
\begin{array}{ll}
\bar{D}_{A}^{2} \phi \circ u+\phi\left(u_{b}^{\prime}(x)+g\left(x, u, u_{T}^{\prime}\right)\right) \leqslant 0, & x \in \Omega, \phi \in K^{*}, \\
\phi\left(a_{0} u\right)+a_{1} \underline{D}_{\nu} \phi \circ u \geqslant 0, & x \in \Gamma, \phi \in K^{*} ;
\end{array}
$$

dabei heisst die Funktion $u$ : $\bar{\Omega} \rightarrow E$ Lösung von (7), (8), wenn sie auf $\bar{\Omega}$ stetig ist, wenn in $\Omega u_{b}^{\prime}$ und $u_{T}^{\prime}$ als schwache Gâteauxsche Ableitungen existieren und wenn (7), (8) gelten.

BEMERKUNG. Es genügt, die Ungleichungen (7), (8) für ein Erzeugendensystem von $K^{*}$ zu verlangen. Insbesondere sind im Fall $E=\mathbf{R}^{m}, K=\{x$ : 
$\left.x_{i} \geqslant 0, i=1, \ldots, m\right\}$ koordinatenweise Ungleichungen für die Gültigkeit von (7), (8) hinreichend.

SATZ 1. Es existiere $U \in \mathcal{E}$, so dass zu jedem $x \in \Omega, z \in E \backslash K, p \in$ $L(T(x), E)$ ein $\phi \in K^{*}$ mit $\phi(z)=-\operatorname{dist}(K, z)$ und

$$
\phi(g(x, z, p)) \geqslant-U\left(x, \operatorname{dist}(K, z),-\phi_{T}(p)\right)
$$

existiert. Dann gilt $u(x) \in K$, d.h. $u(x) \geqslant 0$ für jede Lösung u von (7), (8).

BeweIs. Wir setzen $\eta(x)=\operatorname{dist}(K, u(x))$ und weisen nach, dass $\eta$ alle in der Definition von $\mathcal{E}$ geforderten Eigenschaften besitzt. Zunächst ist $\eta$ stetig auf $\bar{\Omega}$ und nicht negativ. Für diejenigen $x \in \Omega$ mit $\eta(x)=0$ ist sicher $\underline{D}_{A}^{2} \eta(x) \geqslant 0$ und $\underline{D}_{b}^{+} \eta(x) \geqslant 0$. Ist dagegen $\eta(x)>0$, also $u(x) \in E \backslash K$ so existiert nach Voraussetzung ein $\phi \in K^{*}$ mit (9) und $\eta(x)=-\phi(u(x))$; für alle der Norm nach hinreichend kleinen $h \in \mathbf{R}^{n}$ folgt dann aus (3), (4) und (5)

$$
\begin{aligned}
\eta(x+h) & =\operatorname{dist}(K, u(x+h)) \\
& \geqslant \operatorname{dist}(H(\phi), u(x+h))=-\phi(u(x+h)), \\
\eta(x) & =-\phi(u(x)),
\end{aligned}
$$

also für alle hinreichend kleinen $r>0, t \in \mathbf{R}$ und alle $y \in \mathbf{R}^{n}$ mit $|y|=1$

$$
\begin{gathered}
\eta\left(x \pm r A^{1 / 2}(x) y\right)-\eta(x) \geqslant-\phi\left(u\left(x \pm r A^{1 / 2}(x) y\right)-u(x)\right), \\
\eta(x+t b(x))-\eta(x) \geqslant-\phi(u(x+t b(x))-u(x)) .
\end{gathered}
$$

Hieraus folgt $D_{A}^{2} \eta(x)>-\infty, D_{b}^{+} \eta(x)>-\infty$ und, falls $\eta_{T}^{\prime}(x)$ existiert, die Gleichung $\eta_{T}^{\prime}(\bar{x})=-\phi_{T}\left(u_{T}^{\prime}(x)\right)$, was

$$
\begin{aligned}
\underline{D}_{A}^{2} \eta(x) & \geqslant-\left(\bar{D}_{A}^{2} \phi \circ u\right)(x) \geqslant \phi\left(u_{b}^{\prime}(x)+g\left(x, u, u_{T}^{\prime}\right)\right) \\
& \geqslant-\underline{D}_{b}^{+} \eta(x)-U\left(x, \eta(x), \eta_{T}^{\prime}(x)\right)
\end{aligned}
$$

nach sich zieht. Ist schliesslich $x \in \Gamma$ und $\eta(x)=0$, so folgt

$$
a_{0}(x) \eta(x)+a_{1} \bar{D}_{\nu} \eta(x)=\limsup _{k \rightarrow \infty} a_{1}(x)\left(-\eta\left(x_{k}\right)\right) /\left|x-x_{k}\right| \leqslant 0 ;
$$

für $u(x) \in E \backslash K$ hingegen existiert nach dem Satz von Hahn-Banach eine die Mengen $K$ und $B(u(x), \eta(x))$ trennende Hyperebene $H(\phi)$ mit $\phi \in K^{*}$, $\eta(x)=-\phi(u(x)), \quad \eta\left(x_{k}\right) \geqslant \operatorname{dist}\left(H(\phi), u\left(x_{k}\right)\right)=-\phi\left(u\left(x_{k}\right)\right) \quad(k \in \mathbf{N}$ hinreichend gross); damit wird

$$
\begin{aligned}
a_{0} \eta+a_{1} \bar{D}_{\nu} \eta= & -a_{0}(x) \phi(u(x)) \\
& -\limsup _{k \rightarrow \infty} a_{1}(x) \phi\left(u(x)-u\left(x_{k}\right)\right) /\left|x-x_{k}\right| \leqslant 0
\end{aligned}
$$

nach (8).

Es folgt $\eta \equiv 0$, also $u(x) \geqslant 0$ auf $\bar{\Omega}$, wie behauptet.

Folgerung 1. Die Funktionen $v, w: \bar{\Omega} \rightarrow E$ seien stetig, in $\Omega$ mögen $v_{T}^{\prime}$ und $w_{T}^{\prime}$ existieren, und zu jedem $\phi \in K^{*}$ gebe es eine Konstante $K_{\phi}$ mit 


$$
\begin{aligned}
& \underline{D}_{A}^{2} \phi \circ v+\phi\left(v^{\prime} b+f\left(x, v, v_{T}^{\prime}\right)\right) \geqslant K_{\phi} \\
& \geqslant \bar{D}_{A}^{2} \phi \circ w+\phi\left(w^{\prime} b+f\left(x, w, w_{T}^{\prime}\right)\right), \quad x \in \Omega, \\
& \phi\left(a_{0} v\right)+a_{1} \bar{D}_{\nu} \phi \circ v \leqslant K_{\phi} \leqslant \phi\left(a_{0} w\right)+a_{1} \underline{D}_{\nu} \phi \circ w, \quad x \in \Gamma .
\end{aligned}
$$

Die Funktion $f$ sei für festes $(x, z) \in \Omega \times E$ auf $L(T(x), E)$ definiert mit Werten in $E$. Es existiere $U \in \mathcal{E}$, so dass zu jedem $(x, z) \in \Omega \times E$ mit $z \in E \backslash K$ und $p \in L(T(x), E)$ ein $\phi \in K^{*}$ existiert mit $\phi(z)=-\operatorname{dist}(K, z)$ und

$$
\begin{gathered}
\phi\left(f\left(x, v(x)+z, v_{T}^{\prime}(x)+p\right)-f\left(x, v(x), v_{T}^{\prime}(x)\right)\right) \\
\geqslant-U\left(x,-\phi(z),-\phi_{T}(p)\right) .
\end{gathered}
$$

Dann gilt $v \leqslant w$ auf $\bar{\Omega}$.

BeweIs. Wir setzen $u=w-v$ und bemerken, dass $u$ Lösung von (7), (8) mit $g(x, z, p)=f\left(x, v+z, v_{T}^{\prime}+p\right)-f\left(x, v, v_{T}^{\prime}\right)$ ist und dass (13) gerade (9) nach sich zieht. Es folgt $u \geqslant 0$, also $v \leqslant w$ auf $\bar{\Omega}$.

Bemerkungen. (1) Die Konstante $K_{\phi}$ in (11), (12) wird wirklich benötigt, wie das folgende Beispiel zeigt: $n=1, \Omega=(0,1), E=\mathbf{R}^{1}, f \equiv 0, A \equiv 1$, $b \equiv 0, a_{0} \equiv 1, a_{1} \equiv 0$ und $v=\left|x-\frac{1}{2}\right|-\frac{1}{2}, w=2 v$. In diesem Fall ist $D_{A}^{2} v=0$ für $x \neq \frac{1}{2}, D_{A}^{2} v=+\infty$ für $x=\frac{1}{2}$ und $D_{A}^{2} v=D_{A}^{2} w$, aber nicht $v \leqslant w$ in $\Omega$.

(2) Man kann im Beweis zur Folgerung 1 auch $G(x, z, p)=f\left(x, w, w_{T}^{\prime}\right)-$ $f\left(x, w-z, w_{T}^{\prime}-p\right)$ benutzen und muss dann fordern: $\mathrm{Zu} z \in E \backslash K$ existiert $\phi \in K^{*}$ mit $\phi(z)=-\operatorname{dist}(K, z)$ und

$$
\phi\left(f\left(x, w, w_{T}^{\prime}\right)-f\left(x, w-z, w_{T}^{\prime}-p\right)\right) \geqslant-U\left(x,-\phi(z),-\phi_{T}(p)\right) .
$$

Diese Bemerkung ist bei Abschneide- und Fortsetzungsprozessen, wie sie bei Existenzsätzen verwendet werden, von Nutzen.

(3) Über den Zusammenhang zwischen (9) und den in [9], [13] verwendeten Bedingungen an $f$ in Stützpunkten von $K$ wurde in [5] eingegangen.

4. Beispiele. (1) Es sei $n=1, E=\mathbf{R}^{m}$ (Maximumnorm), $K=\{x=$ $\left.\left(x_{1}, \ldots, x_{m}\right): x_{i} \geqslant 0\right\}$ der natürliche Ordnungskegel, $\Omega=(0,1), \Gamma=\{0,1\}$, $A \equiv 1, T(x)=\mathbf{R}^{1}, b \equiv 0, \eta(0)=-1$ und $\nu(1)=1$. In diesem Fall gelten (11), (12), wenn (es ist z.B.

$$
\underline{D}_{A}^{2} v(x)=\liminf _{h \rightarrow 0+} \frac{1}{h^{2}}\{v(x+h)-2 v(x)+v(x-h)\}
$$

komponentenweise, $v_{T}^{\prime}=v^{\prime}$ )

$$
\begin{aligned}
\underline{D}_{A}^{2} v(x)+f\left(x, v, v^{\prime}\right) & \geqslant 0, \quad x \in(0,1), \\
a_{0}(0) v(0)-a_{1}(0) \underline{D}^{+} v & \leqslant 0, \\
a_{0}(1) v(1)+a_{1}(1) \bar{D}^{-} v & \leqslant 0
\end{aligned}
$$

gelten. Dabei ist $a_{0}, a_{1} \geqslant 0$ und $0 \cdot \infty=0$ vorausgesetzt. Entsprechendes gilt für 


$$
\begin{aligned}
\bar{D}_{A}^{2} w(x)+f\left(x, w, w^{\prime}\right) & \leqslant 0, \quad x \in(0,1), \\
a_{0}(0) w(0)-a_{1}(0) \bar{D}^{+} w & \geqslant 0, \\
a_{0}(1) w(1)+a_{1}(1) \underline{D}-w & \geqslant 0 .
\end{aligned}
$$

Anwendung von Satz 2 auf diesen Sachverhalt liefert

Folgerung 2. Für $v, w \in C[0,1] \cap C^{1}(0,1)$ mögen (14) und (15) bestehen, und für $f:(0,1) \times \mathbf{R}^{m} \times \mathbf{R}^{m} \rightarrow \mathbf{R}^{m}$ gelte: Ist $x \in(0,1)$ und $z \in \mathbf{R}^{m} \backslash K$ und $p \in \mathbf{R}^{m}$, so gibt es einen Index $i(z)$ mit $z_{i(z)}=\min \left\{z_{j}\right\}<0$ und

$$
\begin{aligned}
f_{i(z)}(x, v(x)+ & \left.z, v^{\prime}(x)+p\right)-f_{i(z)}\left(x, v(x), v^{\prime}(x)\right) \\
& \left.\geqslant L z_{i(z)}-M \mid p_{i(z)}\right)
\end{aligned}
$$

die Funktion $U(x, s, q)=L s+M|q|$ sei aus $\mathcal{E}$. Dann gilt $v \leqslant w$ in $(0,1)$.

Folgerung 2 ist eine Verallgemeinerung von Resultaten von Heimes [3, Theorem 1, nichttrivialer Teil], Thompson [12] und von Fountain und Jackson [2], vgl. auch Bernfeld-Lakshmikantham [1]. Dabei ist zu beachten, dass für differenzierbares $u$ stets

$$
\underline{D} u^{\prime} \leqslant \underline{D}_{A}^{2} u \leqslant \bar{D}_{A}^{2} u \leqslant \bar{D} u^{\prime} \quad(A \equiv 1)
$$

gilt. $\left(D u^{\prime}(x)=\lim \inf _{h \rightarrow 0}\left[\left(u^{\prime}(x+h)-u^{\prime}(x-h)\right) / 2 h\right]\right.$. Die Forderung $A_{1}$ von Heimes [3] bezüglich $-f$ ist im wesentlichen die Voraussetzung von Folgerung 1 mit $L=0, M$ beliebig.)

Es sei bemerkt, dass für den Fall, dass $f$ nicht von $p$ abhängt, Folgerung 2 sogar für stetige Funktionen $v, w$ gilt und (für $a_{0}=1, a_{1}=0$ ) die Funktion $U(x, s)=L s$ in $\varepsilon$ liegt, falls $L<\pi^{2}$ gilt.

Es folgt ein hinreichendes Kriterium für die Zugehörigkeit einer Funktion $U$ zur Klasse $\mathcal{E}$.

SATZ 2. Existiert zur Funktion $U$, die für jedes $(x, s) \in \Omega \times(0, \infty)$ auf $L(T(x), \mathbf{R})$ definiert ist, eine Schar $\left\{\psi^{(\varepsilon)}, 0<\varepsilon<\infty\right\}$ von auf $\Omega \cup \Gamma$ stetigen Funktionen, die in $\Omega$ differenzierbar sind und für die gilt:

(a) $\mathrm{Zu}$ jeder Funktion $\eta \in C(\bar{\Omega})$ mit $a_{0} \eta(x)+a_{1} \bar{D}_{\nu} \eta(x) \leqslant 0$ auf $\Gamma$ und $\eta \geqslant 0$ in $\Omega, \eta \neq 0$, existiert ein $\varepsilon \in(0, \infty)$ und ein $x_{0} \in \Omega \cup \Gamma$ mit

$$
\psi^{(\varepsilon)}(x)-\eta(x) \geqslant \psi^{(\varepsilon)}\left(x_{0}\right)-\eta\left(x_{0}\right)=0, \quad x \in \Omega
$$

(b) $\bar{D}_{A}^{2} \psi(x)+\psi^{\prime}(x) b(x)+U\left(x, \psi(x), \psi_{T}^{\prime}(x)\right)<0$ in $\Omega, a_{0} \psi(x)+a_{1} \underline{D}_{\nu} \psi(x)$ $>0$ auf $\Gamma$ (für jedes $\psi=\psi^{(\varepsilon)}, 0<\varepsilon<\infty$ )

und ist stets $T(x) \subseteq R(A(x))$, so folgt $U \in \mathcal{E}$.

BEwEIS. Wir betrachten $\eta$ mit den in der Definition von $\mathcal{E}$ beschriebenen Eigenschaften. Ist $\eta \neq 0$, so existiert nach (a) eine Funktion $\psi=\psi^{(\varepsilon)}$ und ein $x_{0} \in \Omega \cup \Gamma \mathrm{mit}$

$$
\psi(x)-\eta(x) \geqslant \psi\left(x_{0}\right)-\eta\left(x_{0}\right)=0, \quad x \in \Omega .
$$

Ist $x_{0} \in \Omega$, so folgt zunächst $\bar{D}_{A}^{2}(\psi-\eta)\left(x_{0}\right) \leqslant \bar{D}_{A}^{2} \psi\left(x_{0}\right)-\underline{D}_{A}^{2} \eta\left(x_{0}\right)<+\infty$, also die Existenz von $\eta_{T}^{\prime}\left(x_{0}\right)$ und die Gleichung $\psi_{T}^{\prime}\left(x_{0}\right)=\eta_{T}^{\prime}\left(x_{0}\right)$ (vgl. [5, 
Hilfssatz 1]). Damit ist wegen $\bar{D}_{A}^{2}(\psi-\eta)\left(x_{0}\right) \geqslant 0, \underline{D}_{b}^{+} \eta\left(x_{0}\right) \leqslant \psi^{\prime}\left(x_{0}\right) b\left(x_{0}\right)$

$$
\begin{aligned}
0 & \leqslant \underline{D}_{A}^{2} \eta\left(x_{0}\right)+\underline{D}_{b}^{+} \eta\left(x_{0}\right)+U\left(x_{0}, \eta\left(x_{0}\right), \eta_{T}^{\prime}\left(x_{0}\right)\right) \\
& \leqslant \bar{D}_{A}^{2} \psi\left(x_{0}\right)+\psi^{\prime}\left(x_{0}\right) b\left(x_{0}\right)+U\left(x_{0}, \psi\left(x_{0}\right), \psi_{T}^{\prime}\left(x_{0}\right)\right)<0
\end{aligned}
$$

nach (b), also $x_{0} \in \Omega$ nicht möglich. Ist aber $x_{0} \in \Gamma$, so folgt

$$
0 \geqslant a_{0} \eta\left(x_{0}\right)+a_{1} \bar{D}_{\nu} \eta\left(x_{0}\right) \geqslant a_{0} \psi\left(x_{0}\right)+a_{1} \underline{D}_{\nu} \psi\left(x_{0}\right)>0,
$$

also ebenfalls ein Widerspruch. Es folgt $\eta \equiv 0$, also $U \in \mathcal{E}$.

Bemerkungen. (1) Ist die Norm in $E$ für $z \neq 0$ im Fréchetschen Sinn differenzierbar, so ist die im Beweis von Satz 1 verwendete Funktion $\eta(x)=$ $\operatorname{dist}(K, u(x))$ in allen Punkten $x$, in denen sie positiv ist, nach $T(x)$ differenzierbar, falls $u$ diese Eigenschaft besitzt, vgl. [6]. In diesem Fall muss über $T(x)$ keine Einschränkung gemacht werden, d.h. in $f$ dürfen alle ersten Ableitungen von $u$ vorkommen. Dieser Fall $\left(E=\mathbf{R}^{m}\right.$, Euklidisch normiert) wurde in [10] und [15] betrachtet.

(2) Wird über die Norm in $E$ weiter nichts vorausgesetzt, so muss (vgl. Satz 2) $T(x) \subseteq R(A(x))$ verlangt werden, d.h. in $f$ darf höchstens nach $R(A(x))$ differenziert werden. Für streng elliptische Gleichungen ist dies kein Verlust an Allgemeinheit.

(3) Für $n=1, A(x) \equiv 0, b(x) \equiv 1, T(x)=\{0\}$ erhält man Ergebnisse aus [13], für $A(x) \equiv 1, b(x) \equiv 0, T(x)=\mathbf{R}$ und $E=\mathbf{R}^{m}$ (Maximumnorm) Verallgemeinerungen von Resultaten aus [3] und [12].

(4) Mit der hier benutzten Methode lassen sich wie in [4] auch Monotoniesätze für (schwach) elliptische Gleichungen auf unbeschränkten Grundgebieten (etwa für das Cauchyproblem bei parabolischen Gleichungen oder für Gleichungen "ohne Anfangswerte") gewinnen. Ausserdem können wie in [4] allgemeinere, nichtlineare Randbedingungen zugelassen werden.

(5) Ist $\Omega$ beschränkt, gilt $U(x, s, q)=L s+M|q|$ und gibt es $\psi \in C(\bar{\Omega}) \cap$ $C^{1}(\Omega), \psi>0$, mit $\bar{D}_{A}^{2} \psi+\psi^{\prime} b+L \psi+M\left|\psi_{T}^{\prime}\right|<0$ in $\Omega, a_{0} \psi+a_{1} \underline{D}_{\nu} \psi>0$ auf $\Gamma$, so genügt $\psi^{(e)}=\varepsilon \cdot \psi$ den Voraussetzungen von Satz 2 .

\section{LITERATUR}

1. S. R. Bernfeld und V. Lakshmikantham, An introduction to nonlinear boundary value problems, Academic Press, New York, 1974.

2. L. Fountain und L. A. Jackson, $A$ generalized solution of the boundary value problem for $y^{\prime \prime}=f\left(x, y, y^{\prime}\right)$, Pacific J. Math. 12 (1962), 1251-1272.

3. K. H. Heimes, Boundary value problems for ordinary nonlinear second order systems, J. Differential Equations 2 (1966), 449-463.

4. R. Lemmert, Über die Invarianz einer konvexen Menge in bezug auf Systeme von gewöhnlichen, parabolischen und elliptischen Differentialgleichungen, Math. Ann. 230 (1977), 49-56.

5. __ Über die Invarianz konvexer Teilmengen eines normierten Raumes in bezug auf elliptische Differentialgleichungen, Comm. Partial Differential Equations (erscheint in).

6. R. Lemmert und P. Volkmann, Ein Invarianzsatz für Systeme von nichtlinearen elliptischen Differentialgleichungen zweiter Ordnung (Manuskript).

7. , Randwertprobleme für gewöhnliche Differentialgleichungen in konvexen Teilmengen eines Banachraumes, J. Differential Equations 27 (1978), 138-143. 
8. M. H. Protter und H. F. Weinberger, Maximum principles in differential equations, Prentice-Hall, Englewood Cliffs, N.J., 1967.

9. R. Redheffer und W. Walter, Flow-invariant sets and differential inequalities in normed spaces, Applicable Analysis 5 (1975), 149-161.

10. Invariant sets for systems of partial differential equations. I. Parabolic equations, Arch. Rational Mech. Anal.

11. R. C. Thompson, Differential inequalities for infinite second order systems and an application to the method of lines, J. Differential Equations 17 (1975), 421-434.

12. Convergence and error estimate for the method of lines for certain nonlinear elliptic and elliptic-parabolic equations, SIAM J. Numer. Anal. 13 (1976), $27-43$.

13. P. Volkmann, Uber die Invarianz konvexer Mengen und Differentialungleichungen in einem normierten Raume, Math. Ann. 203 (1973), 201-210.

14. W. Walter, Differential and integral inequalities, Ergebnisse der Mathematik und ihrer Grenzgebiete, Vol. 55, Springer-Verlag, Berlin and New York, 1970 (Übersetzung aus dem Deutschen).

15. H. F. Weinberger, Invariant sets for weakly coupled parabolic and elliptic systems, Rend. Mat. 8 (1975), 295-310.

Mathematisches Institut I, Universitït Karlsruhe (th), Postfach 6380, 75 Karlsruhe 1, Federal Republic of Germany 